\title{
Computation of Frequencies and Linestrengths for Triatomic Molecules of Astronomical Interest
}

\author{
Steven Miller ${ }^{1}$, Jonathan Tennyson ${ }^{1}$, \\ Hugh R.A. Jones ${ }^{2}$ and Andrew J. Longmore ${ }^{2}$ \\ 1 Department of Physics and Astronomy, University College London, \\ Gower St., London WC1E 6BT, U.K. \\ 2 Royal Observatory Edinburgh, Blackford Hill, Edinburgh EH9 3HJ, U.K.
}

\section{Introduction}

Molecular bands have been used as a diagnostic of spectral type since the 1860's. $\mathrm{TiO}$ was first identified as the dominant feature in optical spectra of cool giants in 1904 (Fowler 1904). Many diatomic molecules have now been included (e.g. Jørgensen \& Larsson 1990) in detailed calculations of stellar opacity and attention is starting to focus on the incorporation of accurate data for triatomics. Triatomic molecules have a much greater density of states than diatomics; bending vibrational bands - generally lower in frequency than bond-stretching modes - may contribute considerably to "filling in" spectral gaps.

From the point of view of quantum chemistry, molecules can access regions which would be described classically as "chaotic" at the temperatures (usually a few thousand degrees) available in cool stellar atmospheres. Molecular transitions associated with such behaviour are to be found, in particular, in the infrared. Astronomically the study of this spectral region has recently benefitted from high resolution, sensitive spectrometers which are now available on many telescopes. Thus there is mutual advantage both for chemical physicists and for astronomers in the sharing of observational and theoretical data for cool star atmospheres.

The dominant red and infrared luminosity of the underlying stellar populations of galaxies is from $M$ giants. The dominant population in terms of number density is from $M$ dwarfs. These $M$ stars emit the bulk of their radiation in the red and infrared where molecules provide the dominant source of opacity in the stellar atmosphere. Detailed understanding of the evolution of $\mathrm{M}$ stars is compromised by the inability to construct model atmospheres without quantitative data for triatomic molecules. Two triatomic molecules are of the utmost importance in stellar atmospheres. For cool O-rich $\mathbf{M}$ stars, particularly the $\mathbf{M}$ dwarfs, the water molecule is the dominant source of opacity in the infrared (Alexander et al. 1989). For C-rich red giant stars, HCN plays an important role (Jørgensen et al. 1985). Accurate potential energy surfaces are available for both molecules.

For water, we have tested four electronic ground-state potential energy surfaces which have been produced in recent years (Carter \& Handy 1987, Halonen \& Carrington 1988, Jensen 1989 and Kauppi \& Halonen 1990). In this paper 
we present only calculations using the Jensen (1989) potential, which was found to be in significantly better agreement with experimental data than the other surfaces, for both vibrational band origins and rotational term values (Fernley et al. 1992. Transition strengths are obtained from an accurate dipole surface computed by Wattson \& Rothman (1993). For HCN we have used a recent potential developed for the whole HCN/HNC system (Gadzy \& Bowman 1991), which puts the barrier to isomerisation at around $17000 \mathrm{~cm}^{-1}$. Band intensities, using a dipole surface valid only for the HCN end of the molecule (Sebald 1991), are presented.

For temperatures applicable to cool star atmospheres (up to $5000 \mathrm{~K}$ ) we consider it will be necessary to compute all levels up to $30000 \mathrm{~cm}^{-1}$ above the ground state. Calculating all transition frequencies and line strengths up to 14000 to $15000 \mathrm{~cm}^{-1}$ (wavelengths longer than $0.7 \mu \mathrm{m}$ ) will cover the near to far infrared spectral region for which these triatomics are likely to be the main source of opacity. In this paper, however, we report only prelimimary calculations covering a much more restricted spectral range.

\section{Ro-vibrational line positions and intensities}

Spectroscopists often use techniques based on perturbation expansions to calculate vibrational levels close to the ground state (e.g. Flaud et al. 1983). This involves fitting measured levels using a perturbational Hamiltonian. The parameters obtained from the fit are then used to predict higher, unobserved, levels. Rotational levels belonging to each vibrational manifold may similarly be computed for moderate angular momentum, J, levels. Such techniques, however, become inappropriate when the vibrational modes involve large amplitude motions and when the angular momentum is such that centrifugal distortion plays a major role.

Additionally, perturbational approaches are based on an idealised model in which rotational and vibrational motions are uncoupled to first order. Rovibrational coupling is then added through coriolis contributions, again considered to be perturbations of the ideal rigid-rotor, harmonic oscillator system. The distribution of line intensities is similarly approximated by assuming there is an intrinsic vibrational band intensity, which is then distributed across the rotational structure using Hönl-London factors (Herzberg 1991).

Present techniques of solving the radiative transfer equation in model atmosphere computations make use of a statistical treatment of the computed monochromatic absorption coefficient, as in for example the Opacity Distribution Functions (Jørgensen \& Larsson 1990). It might be argued that very accurate calculations of molecular data, requiring large amounts of super-computer processing time, are not worthwhile for computing ODFs and that the approach outlined above is sufficient. For many molecules which have large anharmonicity coefficients and centrifugal distortion constants, however, perturbational expansions of the energy levels become divergent at higher ro-vibrational energies and line frequencies calculated from such Hamiltonians may be out by tens or even 
hundreds of $\mathrm{cm}^{-1}$. The water molecule, in particular, is often taken as the classic example for which the perturbation expansion of its rotational motion diverges. For the ground state, the rigid rotor approximation computes the $J=20, K_{a}=20$, level to be some $2000 \mathrm{~cm}^{-1}$ too high; addition of the first correction, the centrifugal distortion, makes the level $\sim 3000 \mathrm{~cm}^{-1}$ too low; the next term in the series causes an error of approximately $+4000 \mathrm{~cm}^{-1}$ (Polyansky 1993). ODFs are known to be sensitive to changes in the monochromatic absorption coefficient at well below this level.

These effects will also cause the distribution of intensity between individual lines using Hönl-London factors to be a poor representation of the actual system. Additionally, effects such as "intensity stealing" can cause whole "dark bands" to become intense; the magnitude of this effect may not be uniform across the entire band, but may depend on individual rotational states. Thus a combination of poor line positions and inappropriately distributed intensity may cause the computed wavelength dependence of the opacity to be very inaccurate. $A$ fortiori, any model atmosphere which predicts synthetic spectra for stars needs to have accurate molecular data as input if significant conclusions about physical conditions within stellar atmospheres are to be drawn.

In the last decade, a number of groups (e.g. Tennyson 1986, Spirko \& Jensen 1985, Carter \& Handy 1986) have developed techniques for calculating triatomic energy levels based on variational techniques. In particular, Sutcliffe \& Tennyson (1986) have produced a ro-vibrational Hamiltonian which is exact within the constraints of the Born-Oppenheimer approximation (which decouples electronic from nuclear motion in a molecule) and the limitations of the electronic potential energy surface available.

The Sutcliffe-Tennyson kinetic energy operator directly relates the cartesian positions of the nuclei of a triatomic molecule to a set of internal co-ordinates comprising two radial co-ordinates and the included angle. Rotation of the molecule-fixed internal co-ordinates in the laboratory frame is carried by the usual Wigner rotational matrices. This operator makes no a priori assumptions about ro-vibrational separation or equilibrium geometry. In addition, the operator includes parameters which relate the radial co-ordinates to the bonds of the molecule in a flexible way, leading to computational advantages, especially for the accurate calculation of high ro-vibrational energy levels. Other features of the method are described elsewhere (Tennyson et al. 1993).

Ro-vibrational wavefunctions may be calculated by two essentially separate techniques. An approach using model basis functions (Finite Basis Representation or FBR) as building blocks has been developed into a suite of "user-friendly" programs, the latest version of which is described in Tennyson et al. (1993). This suite, known as TRIATOM, contains modules to calculate ro-vibrational transition frequencies and linestrengths from dipole moment surfaces as well as synthetic spectra.

A second, more recent, approach has been pioneered by Light and coworkers (see e.g. Bacic \& Light 1989). Known as the Discrete Variable Representation (DVR), this technique calculates wavefunctions on a grid in co-ordinate space (as 
opposed to the FBR method, which can be considered to calculate wavefunctions on a grid in function space). The DVR method is very efficient at building up wavefunctions for high-lying levels. Henderson et al. (1990a,b) have used this method to calculate all the bound vibrational states of $\mathrm{H}_{3}^{+}$(dissociation energy $\left.\sim 36000 \mathrm{~cm}^{-1}\right)$. A program suite using the DVR technique is also now available (Henderson et al. 1993), and additional developments, required to take full account of molecular symmetry, are currently nearing completion.

\section{Water}

\subsection{Ro-vibrational calculations}

At equilibrium, the water molecule has $\mathrm{C}_{2 v}$ symmetry, with an angle of about $105^{\circ}$ between the two $\mathrm{O}-\mathrm{H}$ bonds. However, the molecule can access linear geometries at energies around $12000 \mathrm{~cm}^{-1}$ above the minimum, well below the maximum energy level we consider necessary for cool star opacities. Figure 1 shows a contour plot of the wavefunction of the $162 \mathrm{nd}$ vibrational level of water (calculated using the Jensen potential (1989); $\mathrm{E}=25941 \mathrm{~cm}^{-1}$ ). The $\mathrm{x}$-axis is the inter-bond angle, $\theta$, (in radians) and the $y$-axis the sum of the two bonds. In this representation, 18 nodes may be counted in the $\theta$-co-ordinate, indicating that there are 18 quanta of bend in this level. The wavefunction has appreciable amplitude for almost all values of $\theta$ from $27^{\circ}$ to $180^{\circ}$, showing the large displacements of the bond angle from equilibrium.

We have carried out rigorous testing of the four recent potential energy surfaces mentioned above (Fernley et al. 1992) using the Sutcliffe-Tennyson Hamiltonian. All of them perform reasonably well when compared with experimental data for vibrational levels lying below or just above the energy region at which linear geometries become accessible. The surface due to Jensen (1989) gives a $6.4 \mathrm{~cm}^{-1}$ standard deviation from experiment for the band origins up to $\sim 21100 \mathrm{~cm}^{-1}$ and a $0.14 \mathrm{~cm}^{-1}$ standard deviation from experiment for the rotational term values up to $\mathrm{J}=2$ for the first ten bands.

We have used this potential in an FBR calculation of a limited number of rovibrational energy levels and wavefunctions: for $\mathrm{J}=0$ to 14 , we computed $(\mathrm{J}+1)$ $\times 25$ levels for the states with even parity, and 20 less for those of odd parity; for $\mathrm{J}=15$ to 20,375 even and 355 odd states were produced. This gave a coverage of just over $11000 \mathrm{~cm}^{-1}$ for each J-parity block above the lowest energy in the block; at $\mathrm{J}=20$ the lowest level is $\sim 4400 \mathrm{~cm}^{-1}$ above the $\mathrm{J}=0$ zero point energy.

The present calculations were carried out using Radau co-ordinates (Tennyson et al. 1993) for the radial co-ordinates. This method has been shown to produce very accurate and efficient results for rotationally excited states of $\mathrm{H}_{2} \mathrm{~S}$ and its isotopomers (Miller et al. 1990). Our calculations represent the radial co-ordinate stretching basis functions by Morse-like oscillators, $\Phi(r)$, (Sutcliffe \& Tennyson 1986), up to a total of 17 functions for each radial coordinate, and the bending co-ordinate basis functions by associated Legendre functions, $P_{\lambda}^{k}(\cos \theta)$, with $\lambda \leq 40$. The rotational motion of the molecule is represented by Wigner rotation matrices, $D_{M, k}^{J}(\alpha \beta \gamma)$. The energy of each $\Phi\left(r_{1}\right) \times$ 


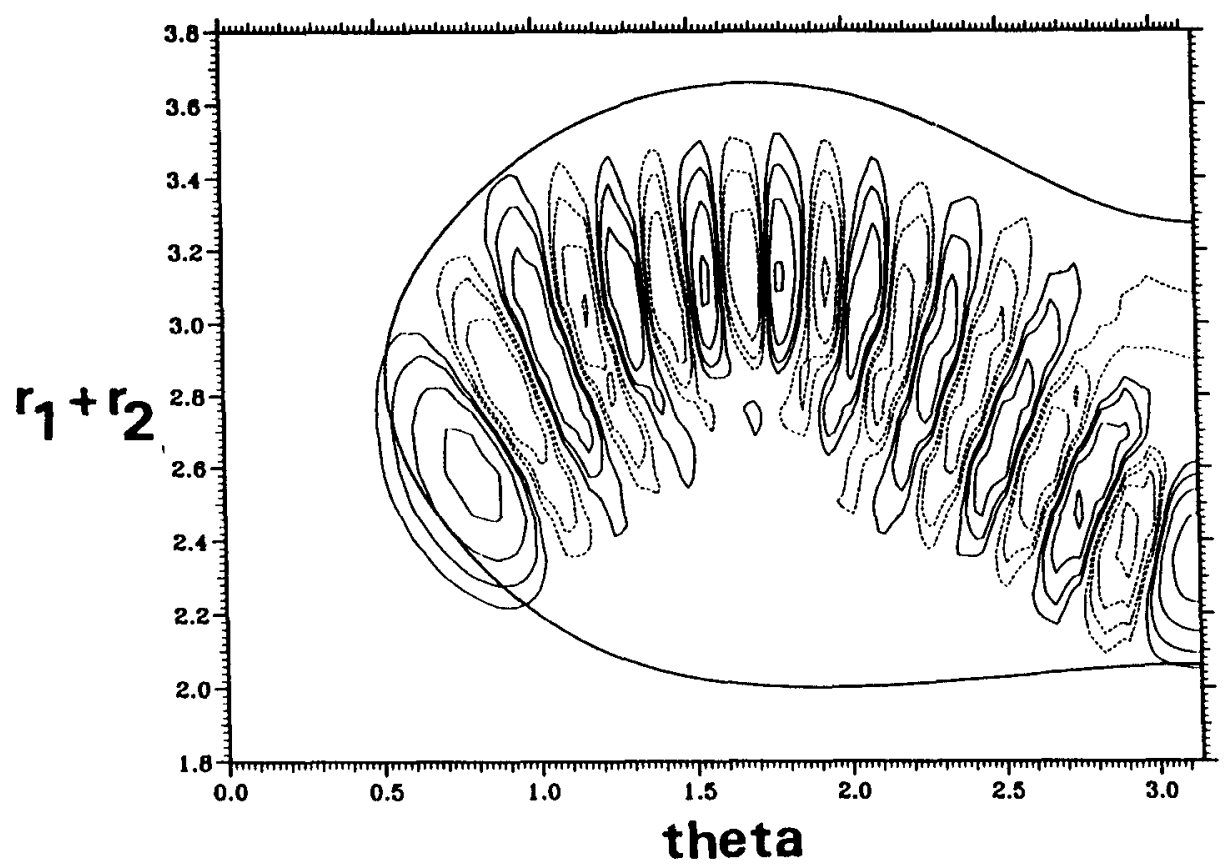

Fig. 1. Contour plot of wavefunction for vibrational level 162

$\Phi\left(r_{2}\right) \times P_{\lambda}^{0}(\cos \theta) \times D_{M, 0}^{0}(\alpha \beta \gamma)$ product basis function was calculated, and the lowest 800 taken to define the intermediate basis functions, $\Phi\left(r_{1}\right) \times \Phi\left(r_{2}\right) \times$ $P_{\lambda}^{k}(\cos \theta) \times D_{M, k}^{J}(\alpha \beta \gamma)$. Of these, a total of $(\mathrm{J}+1) \times 650$ or 9500 for $\mathrm{J} \geq 15$, ordered on the basis of energy, were used in the final ro-vibrational calculation.

Comparison of the 25 lowest $\mathrm{J}=0$ vibrational energy levels with the highly converged DVR calculation of Fernley et al. (1992) showed that the band origins were all converged to within $0.05 \mathrm{~cm}^{-1}$. The rotational levels were also converged to within $0.05 \mathrm{~cm}^{-1}$ for the ground state and fundamentals rising to a maximum of between $5-15 \mathrm{~cm}^{-1}$ for some of the higher lying states. As convergence does not worsen uniformly with level number, many higher lying states are better converged than the stated limit.

Ro-vibrational transition moments were calculated using the dipole moment surface of Wattson \& Rothman (1993). In table 1, we present a comparison between calculated ro-vibrational transition frequencies and relative intensity for transitions involving $\mathrm{J}=0$ and 1 and those listed in the HITRAN (Rothman 1992) database for 19 low-lying levels. Lines are listed relative to the $[\mathrm{J}=1$, $\mathrm{K}_{a}=0, \mathrm{~K}_{c}=1-\mathrm{J}=1, \mathrm{~K}_{a}=1, \mathrm{~K}_{c}=0$ ] (101-110 in Table 1) transition of the $\nu_{2}$ bending fundamental, which has a calculated intensity of $2.60 \times 10^{-19}$ $\mathrm{cm} /$ molecule in good agreement with the measured value of $2.47 \times 10^{-19} \mathrm{~cm} / \mathrm{mol}$. For these transitions, the agreement with experiment is reasonable. The one serious disagreement is with the $\nu_{3}-\nu_{2}$ difference band, which our calculation 
Table 1. $\mathrm{H}_{2} \mathrm{O}$ : observed and calculated frequencies and relative intensities

\begin{tabular}{|c|c|c|c|c|c|}
\hline Band & Transition & $\omega_{\text {obs }}$ & $I_{o b s}^{a}$ & $\omega_{\text {cal }}$ & $\mathrm{I}_{c a l}^{a}$ \\
\hline \multirow[t]{2}{*}{$000-000$} & $110-101$ & 18.58 & $.213(0)$ & 18.53 & $.200(0)$ \\
\hline & $111-000$ & 37.14 & $.204(0)$ & 37.09 & $.191(0)$ \\
\hline \multirow[t]{2}{*}{ 010-010 } & $110-101$ & 21.95 & $.122(-3)$ & 21.91 & $.129(-3)$ \\
\hline & $111-000$ & 40.22 & $.980(-4)$ & 40.18 & $.103(-3)$ \\
\hline \multirow[t]{2}{*}{$020-020$} & $110-101$ & 26.48 & $.583(-7)$ & 26.40 & $.101(-6)$ \\
\hline & $111-000$ & 44.46 & $.467(-7)$ & 44.39 & $.687(-7)$ \\
\hline \multirow[t]{2}{*}{$010-000$} & $101-110$ & 1576.19 & $.100(+1)$ & 1575.83 & $.100(+1)$ \\
\hline & $111-000$ & 1634.97 & $.235(0)$ & 1634.52 & $.226(0)$ \\
\hline \multirow[t]{2}{*}{$020-010$} & $101-110$ & 1534.93 & $.822(-3)$ & 1535.81 & $.977(-3)$ \\
\hline & $111-000$ & 1601.35 & $.232(-3)$ & 1602.10 & $.219(-3)$ \\
\hline \multirow[t]{2}{*}{$030-020$} & $101-110$ & 1488.67 & $.462(-6)$ & 1489.29 & $.858(-6)$ \\
\hline & $111-000$ & 1565.84 & $.140(-6)$ & 1566.23 & $.189(-6)$ \\
\hline \multirow[t]{2}{*}{$020-000$} & $101-110$ & 3133.07 & $.591(-2)$ & 3133.54 & $.610(-2)$ \\
\hline & $111-000$ & 3196.09 & $.150(-2)$ & 3196.44 & $.150(-2)$ \\
\hline \multirow[t]{2}{*}{$030-010$} & $101-110$ & 3051.07 & $.660(-5)$ & 3051.50 & $.759(-5)$ \\
\hline & $111-000$ & 3122.72 & $.172(-5)$ & 3123.94 & $.190(-5)$ \\
\hline \multirow[t]{2}{*}{$030-000$} & $101-110$ & 4648.21 & $.143(-4)$ & 4649.23 & $.346(-4)$ \\
\hline & $111-000$ & 4717.47 & $.453(-5)$ & 4718.28 & $.977(-5)$ \\
\hline \multirow[t]{2}{*}{$040-000$} & $101-110$ & 6155.39 & $.294(-6)$ & 6115.64 & $.114(-6)$ \\
\hline & $111-000$ & 6194.79 & $.813(-7)$ & 6194.61 & $.300(-7)$ \\
\hline \multirow[t]{2}{*}{$100-000$} & $101-110$ & 3638.08 & $.303(-1)$ & 3637.63 & $.323(-1)$ \\
\hline & $111-000$ & 3693.29 & $.623(-2)$ & 3892.78 & $.597(-2)$ \\
\hline \multirow[t]{2}{*}{$200-000$} & $101-110$ & 7182.15 & $.433(-2)$ & 7183.51 & $.514(-2)$ \\
\hline & $111-000$ & 7236.75 & $.106(-2)$ & 7238.09 & $.110(-2)$ \\
\hline \multirow[t]{2}{*}{$001-000$} & $111-110$ & 3749.33 & $.656(0)$ & 3749.43 & $.510(0)$ \\
\hline & $101-000$ & 3779.49 & $.171(0)$ & 3779.57 & $.149(0)$ \\
\hline \multirow[t]{2}{*}{$002-000$} & $101-110$ & 7425.44 & $.317(-3)$ & 7426.05 & $.384(-3)$ \\
\hline & $111-000$ & 7479.66 & $.112(-3)$ & 7479.70 & $.144(-3)$ \\
\hline \multirow[t]{2}{*}{$101-000$} & $111-110$ & 7242.44 & $.555(-1)$ & 7243.71 & $.258(-1)$ \\
\hline & $101-000$ & 7273.13 & $.152(-1)$ & 7274.24 & $.760(-2)$ \\
\hline \multirow[t]{2}{*}{$100-010$} & $101-110$ & 2039.95 & $.140(-4)$ & 2039.90 & $.118(-4)$ \\
\hline & $111-000$ & 2098.55 & $.400(-5)$ & 2098.43 & $.319(-5)$ \\
\hline \multirow[t]{2}{*}{$001-010$} & $111-110$ & 2151.19 & $.228(-4)$ & 2151.70 & $.152(-7)$ \\
\hline & $101-000$ & 2184.75 & $.660(-5)$ & 2185.23 & $.757(-8)$ \\
\hline \multirow[t]{2}{*}{$110-010$} & $101-110$ & 3617.89 & $.115(-4)$ & 3617.73 & $.112(-4)$ \\
\hline & $111-000$ & 3679.41 & $.238(-5)$ & 3679.22 & $.189(-5)$ \\
\hline \multirow[t]{2}{*}{$011-010$} & $111-110$ & 3729.26 & $.275(-3)$ & 3730.63 & $.276(-3)$ \\
\hline & $101-000$ & 3760.13 & $.733(-4)$ & 3761.41 & $.820(-4)$ \\
\hline
\end{tabular}

Powers of ten in brackets. 
predicts to be four orders of magnitude weaker than is observed.

One limitation of the present calculations is that we were not able to use the properly symmetrised wavefunctions necessary to account automatically for spin statistics. For the construction of low temperature spectra, it is clear that this is a serious problem; agreement with experiment can only be achieved by handlabelling ortho and para levels. At temperatures above about $500 \mathrm{~K}$, however, the mast important ortho and para transitions (nearly) coincide and spin statistics may be ignored to a good approximation.

\subsection{Comparison with M-dwarf spectra}

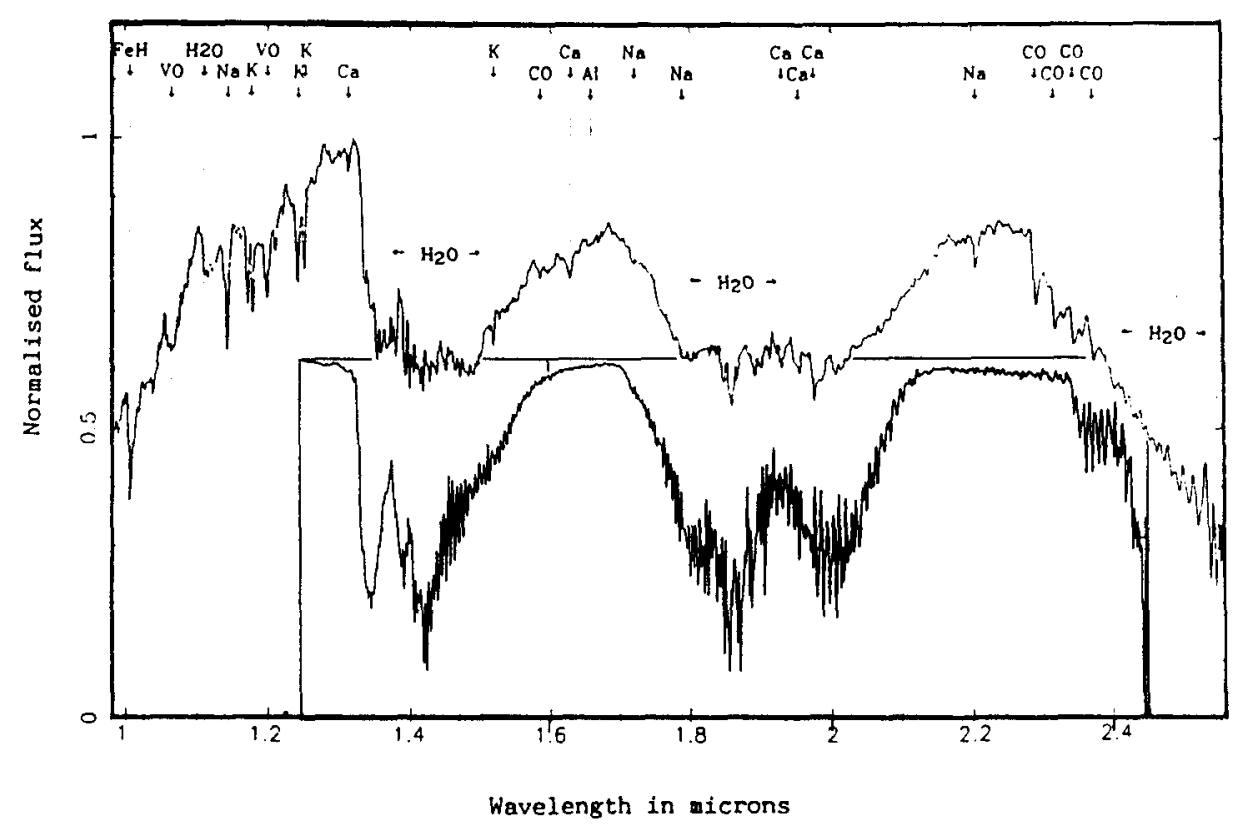

Fig. 2. Comparison of spectrum of VB10 (upper trace) with calculation (lower boxed trace)

The infrared spectra of cool dwarf stars are dominated by strong water absorption features. Jones et al. (1993a) have data on a number of M-dwarfs which 
show that it should be possible to derive an independent check on the temperatures derived from model atmosphere codes (e.g. Allard et al. 1993) by fitting the spectra of these water absorptions to accurate molecular data. The present calculation is too limited, particularly in terms of the maximum $\mathbf{J}$ cutoff, to be able to do this with certainty. But it is possible to test both the ability of the calculated spectrum to reproduce the overall features of the stellar spectrum and to see how sensitive this is to variations in the temperature.

In Fig. 2, we present the spectrum of the M-dwarf VB10 from 1 to $2.5 \mu \mathrm{m}$. Allard et al. (1993) found that the best fit to the observed spectrum was obtained by use of a model atmosphere with an effective temperature of 2800 . The spectrum shown was produced by combining spectra obtained on June 20, 1991 and May 5, 1992 using the cooled grating spectrometer CGS4 on the United Kingdom InfraRed Telescope (Jones et al. 1993a). The spectral range displayed shows two strong absorption features, centred on 1.4 and $1.9 \mu \mathrm{m}$ (as well as the commencement of another at $2.4 \mu \mathrm{m}$ ). In the insert box we show the absorption spectrum (assuming an optically thin gas) calculated for a temperature of $3000 \mathrm{~K}$, with individual lines computed as a Gaussian profile of full-width half-maximum of about half the CGS4 instrument function to show up the calculated spectral detail. No attempt to model the continuum present in VB10 has been made.

The calculated spectrum shows clearly the main features of that of VB10; in particular, the sharp band head at $1.34 \mu \mathrm{m}$ is well reproduced. Analysis of the calculated spectrum shows that the $1.4 \mu \mathrm{m}$ absorption is due, mainly, to the $\nu_{1}+\nu_{3}$ combination band, with weaker contributions from $2 \nu_{1}, 2 \nu_{3}, 2 \nu_{2}+\nu_{1}$ and $2 \nu_{2}+\nu_{3}$, as well as several hot-band transitions. The longer wavelength absorption feature results mainly from the $\nu_{2}+\nu_{1}$ and $\nu_{2}+\nu_{3}$ combination bands, again with a large number of hot-band lines.

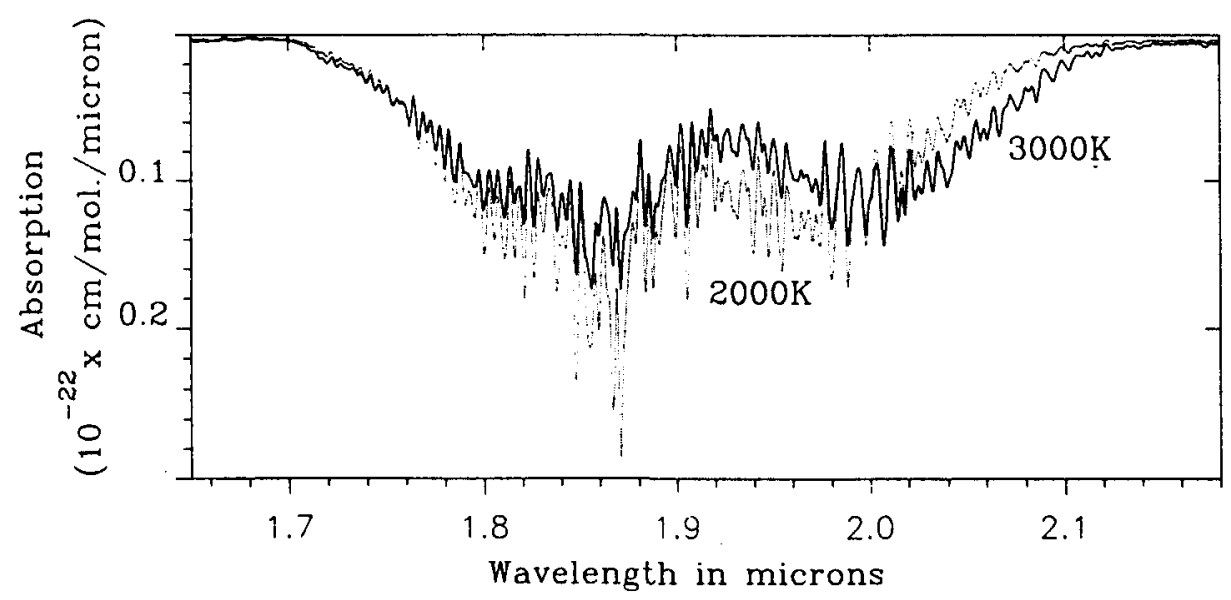

Fig. 3. Temperature sensitivity of calculated $\nu_{2}+\nu_{1} / \nu_{3}$ spectral region: $\mathrm{T}=3000 \mathrm{~K}$ full line; $\mathrm{T}=2000 \mathrm{~K}$ broken line 
In Fig. 3, the calculated absorption spectra in the $\nu_{2}+\nu_{1} / \nu_{3}$ region for $3000 \mathrm{~K}$ and $2000 \mathrm{~K}$ are shown. Both show the $\mathrm{R}$ and $\mathrm{P}$ branch peaks. The spectra show sensitivity to temperature: the $2000 \mathrm{~K}$ (dotted line) spectrum shows a sharper $R$ branch peak, and the maximum in the $P$ branch is at shorter wavelengths (corresponding to lower values of $\mathrm{J}$ ); the $3000 \mathrm{~K}$ spectrum (full line) shows considerably more intensity in the wings, particularly in the $\mathrm{P}$ branch, with the maximum shifted to longer wavelengths (higher $\mathrm{J}$ values).

\section{GL406 21ST ANO 23RD}

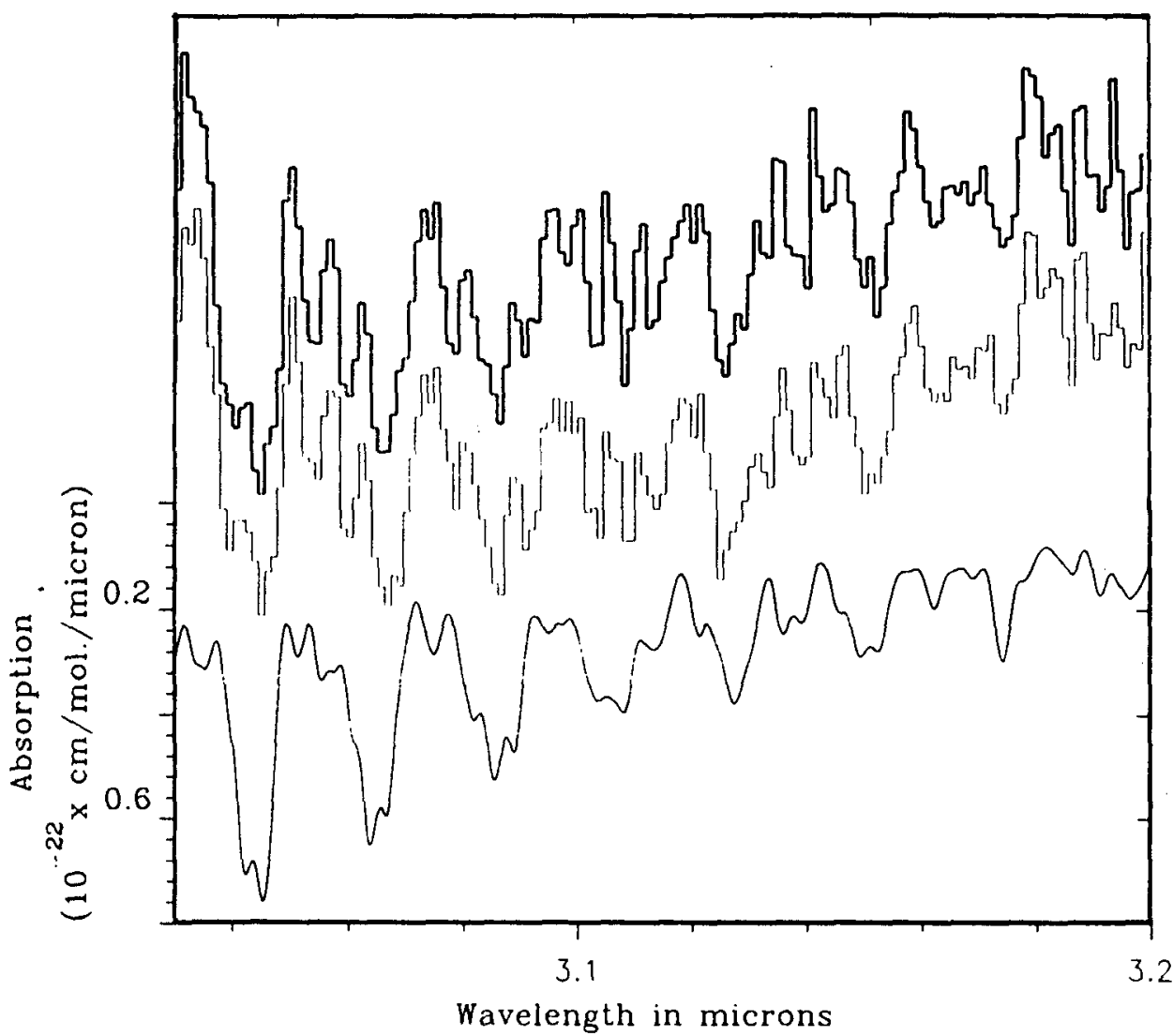

Fig. 4. Comparison of spectrum of GL406 for April 21, 1993 (top trace) and April 23, 1993 (middle trace) with calculation (bottom trace)

More recently, Jones et al. (1993b) have obtained spectra in the 2.85 to 3.4 micron region, corresponding to the $\mathrm{P}$ branch of the $\nu_{3}$ and the $(\sim 20 \times$ weaker $)$ $\nu_{1}$ bands, for a number of M-dwarf stars. One part of these observations taken on 21st April 1993 for the M dwarf GL406 is shown in Fig. 4. Although this spectrum 
was taken by observing through atmospheric water vapour at $250 \mathrm{~K}$ the effect of atmospheric transmission is corrected by dividing the observed spectrum by an appropriate standard star very close in airmass to the target object. The observations were repeated on a subsequent night to check the cancellation of atmospheric features and the reproducibility was found to be excellent. Below these two traces we plot the spectrum calculated at $3000 \mathrm{~K}$; once more the general agreement with the stellar spectrum is very good.

\subsection{Future work}

The calculations presented here for water may be used for astrophysical purposes with four main restrictions:

1) The limited range of energies covered means that total opacity will be underestimated. This effect will become increasingly important at shorter wavelengths and for higher temperatures;

2) The convergence of the energy levels, which becomes generally - though not uniformly - worse with higher vibrational and rotational states, means that opacity is not distributed accurately throughout the band. However, the present calculation will be a considerable improvement on any assuming the rigid rotor approximation (for the same $\mathbf{v}, \mathbf{J}$ states). Moreover, transition frequencies which depend on the difference between energy levels, as opposed to the individual energies themselves, are less affected by the problem of convergence since there is a certain amount of cancellation of errors ;

3) The absence of spin statistics means that both the ro-vibrational partition function and the detailed intensity distribution will be incorrect. Dependent on the resolution required, this problem will probably only be important for temperatures below $500 \mathrm{~K}$, as has been shown to be the case for $\mathrm{H}_{3}^{+}$(Sidhu et al. 1992). Systematic tests to determine how low in temperature one can reliably go are in progress;

4) The Jensen potential, while very good, is not perfect in its prediction of vibrational band origins and rotational term values (Fernley et al. 1992). Similarly, the Wattson dipole surface has some short-comings (see Table 1).

None of these problems is insuperable, given time - human and computer and ingenuity. In collaboration with several co-workers, we have a program to remedy the current defects:

1) A new set of calculations is planned to cover band origins up to $30000 \mathrm{~cm}^{-1}$ and angular momentum states up to 55, at which value of $\mathrm{J}$ the lowest energy level is estimated to be approximately $30000 \mathrm{~cm}^{-1}$ above the zero point energy;

2) The present calculation has been carried out using an FBR and has been designed to fit into 10 megawords of memory. Convergence problems may be almost totally overcome (to within $1 \mathrm{~cm}^{-1}$ ) by switching to the DVR, probably without the need to use more memory;

3) New program developments not only mean that spin statistics can be explicitly included in the calculation but that computational efficiency can be enhanced by a priori excluding spin-forbidden transitions; 
4) There is already a program underway to modify the Jensen potential; preliminary results show a significant improvement in both band origins and rotational term values (Paulse \& Tennyson 1993). Other work is underway to improve the dipole surface.

\section{The HCN/HNC system}

\subsection{DVR calculations for $\mathrm{J}=0$}

Until recently, ro-vibrational calculations of the $\mathrm{HCN} / \mathrm{HNC}$ system have made use of an empirically fitted potential energy surface due to Murrell et al. (1982) (MCH) (Bacic \& co-workers 1986, 1987, 1990). The MCH surface, however, considerably underestimates the barrier to isomerisation $\left(E_{i s o m}=12168 \mathrm{~cm}^{-1}\right)$ and Gazdy \& Bowman (1991) fitted a new surface, based on the 1982 potential, which incorporated the $a b$ initio saddle point $\left(E_{i s o m}=\sim 16500 \mathrm{~cm}^{-1}\right)$. The production of the final surface, which they labelled MCH-M3, also involved scaling the C$\mathrm{H}$ and $\mathrm{C}-\mathrm{N}$ stretching terms. The agreement between calculated and observed vibrational band origins was considerably improved by the MCH-M3 potential, especially for levels above $10000 \mathrm{~cm}^{-1}$. Although a new a $b$ initio surface is now available (Bentley et al. 1992), MCH-M3 is still the global potential which gives the best representation of existing experimental vibrational data.

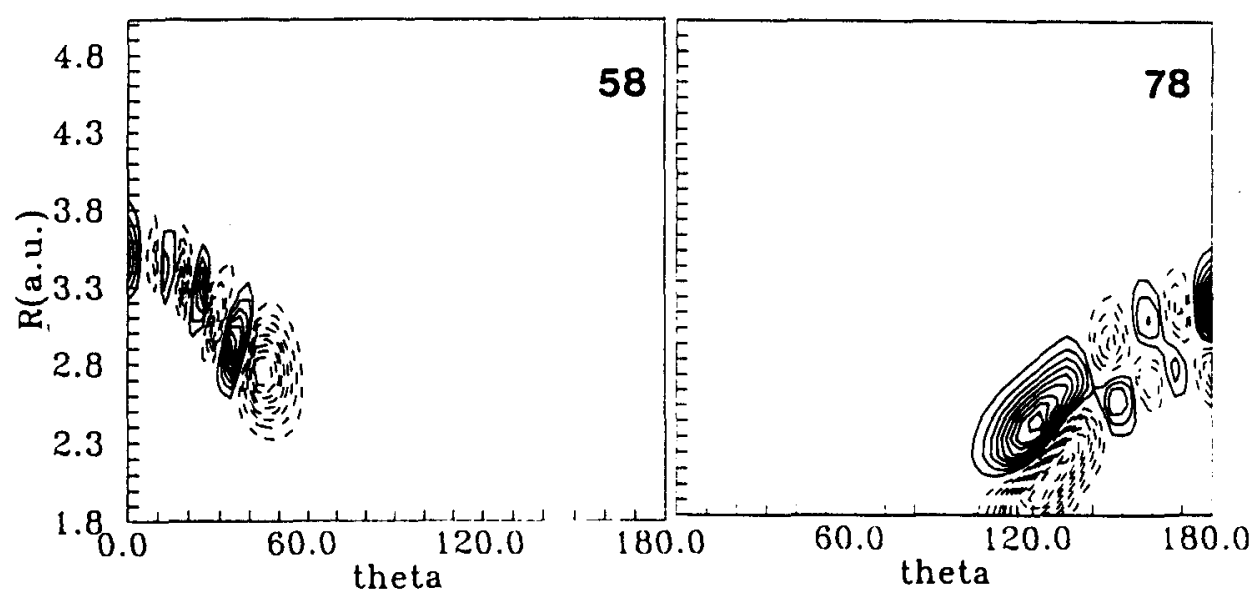

Fig. 5. Contour plots of $R_{H-C N}$ versus $\cos (\theta)$ for vibrational levels $58\left(\mathrm{E}=9585 \mathrm{~cm}^{-1}\right)$ and $78\left(\mathrm{E}=10630 \mathrm{~cm}^{-1}\right)$ 
To our knowledge, no global dipole surface for the HCN/HNC system has been published. A recent $a b$ initio dipole, expressed in terms of the bending co-ordinate and the $\mathrm{C}-\mathrm{H}$ and $\mathrm{C}-\mathrm{N}$ stretches, and valid for $\mathrm{H}-\mathrm{C}-\mathrm{N}$ angles up to $90^{\circ}$, has been produced by Sebald (1991). For band origins below $15000 \mathrm{~cm}^{-1}$, this surface gives a fair representation of the measured band intensities when coupled with the corresponding potential energy surface.

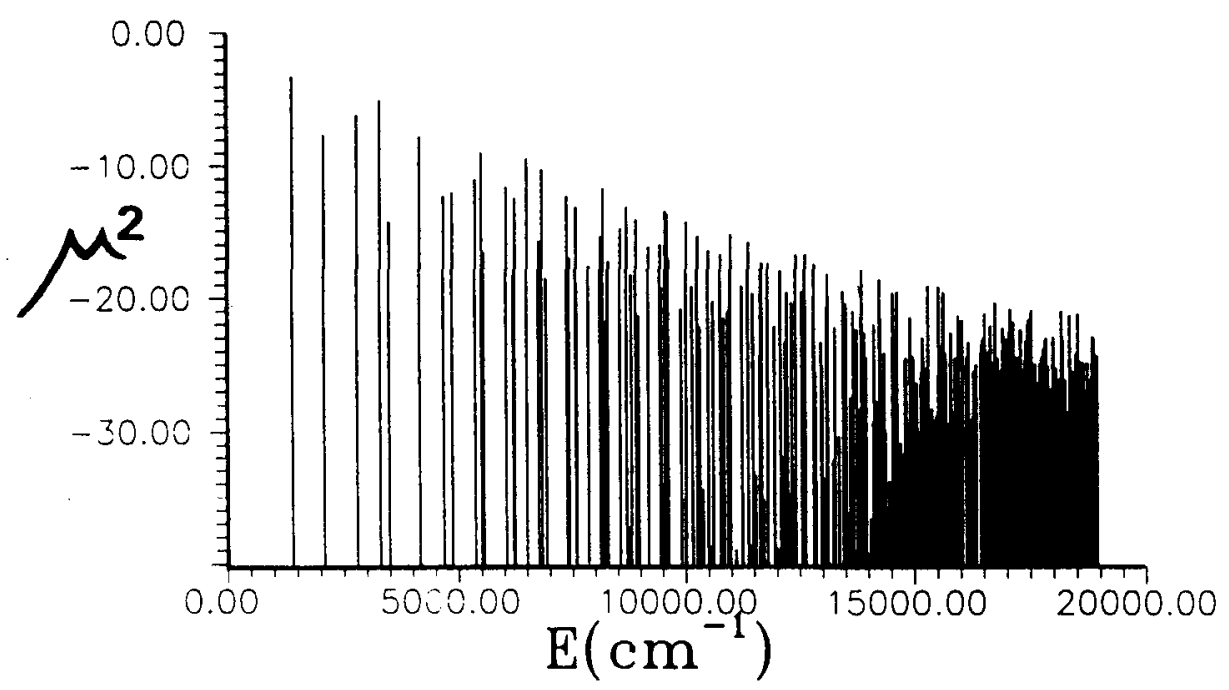

Fig. 6. $\log _{10} \mu^{2}$ versus transition energy for $\mathrm{HCN}$

We have carried out preliminary calculations of the lowest $500 \mathrm{~J}=0$ vibrational levels using the MCH-M3 potential, which extend up to $20000 \mathrm{~cm}^{-1}$ above the minimum. The calculation, perfomed in scattering co-ordinates (Tennyson 1993) was done using a DVR in which intermediate two-dimensional $r_{1}, r_{2}$ gridpoints, with energies below $25000 \mathrm{~cm}^{-1}$, were used to generate a final basis-grid of $2000 r_{1}, r_{2}, \theta$ poin ts to be diagonalised (Henderson et al. 1993). By comparing this calculation with one that used only 1500 final grid-points, convergence at the top of the energy range was estimated to be between 5 and $10 \mathrm{~cm}^{-1}$. We have also calculated $\mathbf{J}=0$ band dipoles using the Sebald dipole for the HCN transitions.

Details of this work and our results will be published in full elsewhere (Seidel et al. 1993). It is clear that - as in the case of water - very large amplitude motions will be important in calculating levels necessary to model stellar atmospheres. Figure 5 shows contour plots for wavefunctions for two energy levels around $10000 \mathrm{~cm}^{-1}$ above the zero point energy. Level 58 shows seven quanta of $\nu_{2}$ for the HCN conformer; level 78 is assigned to the HNC conformer, with four 
quanta of $\nu_{2}$ and one of $\mathrm{N}-\mathrm{H}$ stretch. Although both states are well below the saddle point, large amplitude bending is apparent.

In Fig. 6 , we show the band strengths, in terms of $\mu_{b}^{2}$, for the HCN conformer up to $18000 \mathrm{~cm}^{-1}$. As expected, band strength declines with increasing frequency. At higher energies there is a bath of very low intensity bands, with several more intense transitions, a feature noted by Le Sueur et al. (1993) for $\mathrm{H}_{3}^{+}$. It is clear that the combination of increasing density of states at high energies and the existence of several "intense" transitions means that $\mathrm{HCN} / \mathrm{HNC}$ ro-vibrational opacity will extend right through the 0.5 to $1 \mu \mathrm{m}$ region.

\subsection{Future calculations}

We intend to carry out a similar project to that outlined for the water molecule. Calculations are already underway to extend the Sebald dipole to the HNC conformer of the system (Botschwina 1993), and the $a b$ initio surface will then need to be checked against experimental data (see Lehmann \& Romanini this volume). Using the MCH-M3 surface, our calculations will automatically produce levels for HNC mixed in with those for HCN. For energies sufficiently high above the barrier to isomerisation this is clearly a correct procedure; however, HNC transitions have apparently not been identified in cool star atmospheres (Jørgensen 1993) and it may be necessary to implement some form of separation between the two conformers at lower energies.

\section{Conclusions}

The development of powerful techniques for the computation of large number of ro-vibrational transitions has fortuitously coincided with the advent of highresolution, sensitive infrared astronomical spectrometers. Our preliminary calculations for $\mathrm{H}_{2} \mathrm{O}$ transitions up to $\mathrm{J}=20$ provide a satisfactory fit to the observed features in $\mathrm{M}$ dwarfs both at low resolution from 1.3 to 2.5 microns and at higher resolution from 2.85 to 3.05 microns. For molecules of fundamental astronomical and chemical interest, such as water and $\mathrm{HCN} / \mathrm{HNC}$, it is both feasible and worthwhile to undertake major computational projects to produce the necessary molecular data.

Acknowledgements. The work presented is a progress report of long-term detailed studies involving a large number of co-workers. Oleg Polyansky, John Fernley and Nic Fulton are contributing to the work on the water molecule, and are gratefully acknowledged for their contribution. Luis Seidel, Rosa Benito, Florentino Borondo and James Henderson are thanked for their work on HCN/HNC. Richard Jameson and Matthew Mountain are valued collaborators in the observation of cool star spectra. The U.K. SERC and British Council and the Spanish Accion Integrada provided financial resources for this work. The staff of UKIRT are thanked for their help in obtaining the stellar spectra included. 


\section{References}

Alexander D.R., Augason G.C., Johnson H.R., 1989, Ap. J., 345, 1014

Allard F., Jones H.R.A., Longmore A.J., 1993, Astrophys. J., in preparation

Bacic Z., Light J.C., 1986, J. Chem. Phys., 85, 4594

Bacic Z., Light J.C., 1987, J. Chem. Phys., 86, 3065

Bacic Z., Light J.C., 1989, Ann. Rev. Chem. Phys., 40, 469

Bentley J.A., Bowman J.M., Gazdy B., Lee T.J., Dateo C.E., 1992, Chem. Phys. Lett., 198, 563

Botschwin a P., 1993, private communication.

Carter S., Handy N.C., 1986, Comp. Phys. Reports, 5, 115

Carter S., Handy N.C., 1987, J. Chem. Phys., 87, 4294

Fernley J.A., Miller S., Tennyson J., 1992, Astron. Astrophys., in press

Flaud J.-M., Camy-Peyret C., Johns J.W.C., 1983, Can. J. Chem., 61, 1462

Fowler A., 1904, Proc. R. Soc., 73, 219

Gadzy B., Bowman J.M., 1991, J. Chem. Phys., 95, 6309

Halonen L., Carrington T. Jnr., 1988, J. Chem. Phys., 88, 4171

Henderson J.R., Tennyson J., 1990a, Chem. Phys. Lett., 173, 133

Henderson, J.R., Tennyson, J., Sutcliffe, B.T., 1990b, J. Chem. Phys., 98, 7191

Henderson J.R., Le Sueur C.R., Tennyson J., 1993, Comp. Phys. Commun., 75, 379

Herzberg G., 1991, Molecular Spectra and Molecular Structure, III, Krieger, 226

Jensen P., 1989, J. Mol. Spectrosc., 133, 438

Jones H.R.A., Longmore A.J, Jameson R.F., Mountain C.M., 1993, Mon. Not. Roy. Astr. Soc., submitted

Jones H.R.A., Longmore A.J., Miller S., Tennyson J., 1993, Astrophys. J., in prep.

Jørgensen U.G., Almlöf J., Gustaffson B., Larsson M., Siegbahn P., 1985, J. Chem. Phys., 83, 3034

Jørgensen U.G., Larsson M., 1990, Astron. Astrophys., 238, 424

Jørgensen U.G., 1993, private communication

Kauppi E., Halonen L., 1990, J. Chem. Phys., 94, 5799

Miller S., Tennyson J., Rosmus P., Senekowitsch J., Mills I.M., 1990, J. Mol. Spectrosc., 143, 61

Mladenovic M., Bacic Z., 1990, J. Chem. Phys., 93, 3039

Murrell J.N., Carter S., Halonen L.O., 1982, J. Mol. Spectrosc., 93, 307

Paulse J.D., Tennyson J., 1993, 48th Ohio State University Symposium on Molecular Spectroscopy, Abstract WE12

Polyansky O.L., 1993, private communication

Rothman L.S., et al., 1992, J. Quant. Spectrosc. Radiat. Transfer, 48

Sebald P., 1991, PhD. Thesis, Universitat Kaiserslautern

Seidel L., Benito R.M., Borondo F., Miller S., Henderson J.R., Tennyson J., 1993, in preparation

Sidhu K.S., Miller S., Tennyson J., 1992, Astron. Astrophys., 255, 453

Spirko V., Jensen P., Bunker P.R., Cejchan A., 1985, J. Mol. Spectrosc., 112, 183

Le Sueur C.R., Henderson J.R., Tennyson J., 1993, Chem. Phys. Lett., 206, 429

Sutcliffe B.T., Tennyson J., 1986, Mol. Phys., 58, 1053

Tennyson J., 1986, Comp. Phys. Reports, 4, 1

Tennyson J., Miller S., Henderson J.R., 1993, in Methods in Computational Chemistry, 4, (Plenum, New York 1993), 91

Tennyson J., Miller S., Le Sueur C.R., 1993, Comp. Phys. Commun., 75, 339

Wattson R.B., Rothman L.S., 1993, J. Quant. Spectrosc. Radiat. Transfer, in press 